\title{
Excited state structure of 4-(dimethylamino)benzonitrile studied by femtosecond mid-infrared spectroscopy and ab initio calculations
}

\author{
C. Chudoba ${ }^{\text {a }}$, A. Kummrow ${ }^{\text {a, }}$, J. Dreyer ${ }^{\text {a }}$, J. Stenger ${ }^{\text {a }}$, E.T.J. Nibbering ${ }^{\text {a }}$, \\ T. Elsaesser ${ }^{a}$, K.A. Zachariasse ${ }^{b}$ \\ ${ }^{a}$ Max-Born-Institut für Nichtlineare Optik und Kurzzeitspektroskopie, Max-Born-Strasse 2A, 12489 Berlin, Germany \\ ${ }^{\mathrm{b}}$ Max-Planck-Institut für Biophysikalische Chemie, Spektroskopie und Photochemische Kinetik, 37070 Göttingen, Germany
}

Received 13 April 1999; in final form 24 June 1999

\begin{abstract}
Combining femtosecond transient vibrational spectroscopy and high-level calculations is a powerful tool in the determination of excited-state structures. Striking differences in the experimental vibrational pattern of the locally excited states of 4-(dimethylamino)benzonitrile (DMABN) and 4-aminobenzonitrile (ABN) are explained on the basis of molecular structures obtained from ab initio complete-active-space self-consistent-field (CASSCF) calculations, giving evidence for a strong sensitivity of the molecular structure on modest changes in the substituents. The $4.0 \mathrm{ps}$ charge-transfer time for DMABN in acetonitrile is resolved for the first time by tracking the downshifted $\mathrm{C} \equiv \mathrm{N}$ stretching mode. (C) 1999 Elsevier Science B.V. All rights reserved.
\end{abstract}

\section{Introduction}

Charge-transfer (CT) reactions are among the most important processes in chemistry and biology. Intramolecular charge transfer (ICT) in electronically excited states is conveniently studied with spectroscopic techniques. Over the past decades 4(dimethylamino)benzonitrile (DMABN, Fig. 1) has received continuous interest from experimentalists [1-9] and theoreticians [10] alike. The striking feature of this donor-acceptor compound is the occurrence of dual fluorescence in polar solvents reflecting the existence of two distinct excited-state

\footnotetext{
* Corresponding author. Fax: +49-30-63921429; e-mail: kummrow@mbi-berlin.de
}

intermediates. The normal fluorescence band $\left(L_{b}\right.$ type) has been assigned to a moderately polar locally excited state (LE), whereas the red-shifted anomalous band ( $L_{a}$-type) has been shown to originate from a highly polar CT state [2-9]. In contrast, 4-aminobenzonitrile (ABN, Fig. 1) exhibits only normal fluorescence regardless of solvent polarity [6-8].

Despite major experimental efforts by means of a variety of absorption and emission techniques [1-9], conclusive evidence for excited-state structures in condensed phase is still lacking. Rotationally resolved gas-phase spectroscopy has provided some indication of structural changes in the LE states of $\mathrm{ABN}$ and DMABN [11-13]. Further information on LE structures has been obtained by ab initio CI-singles [14] and semi-empirical calculations [15-17]. 


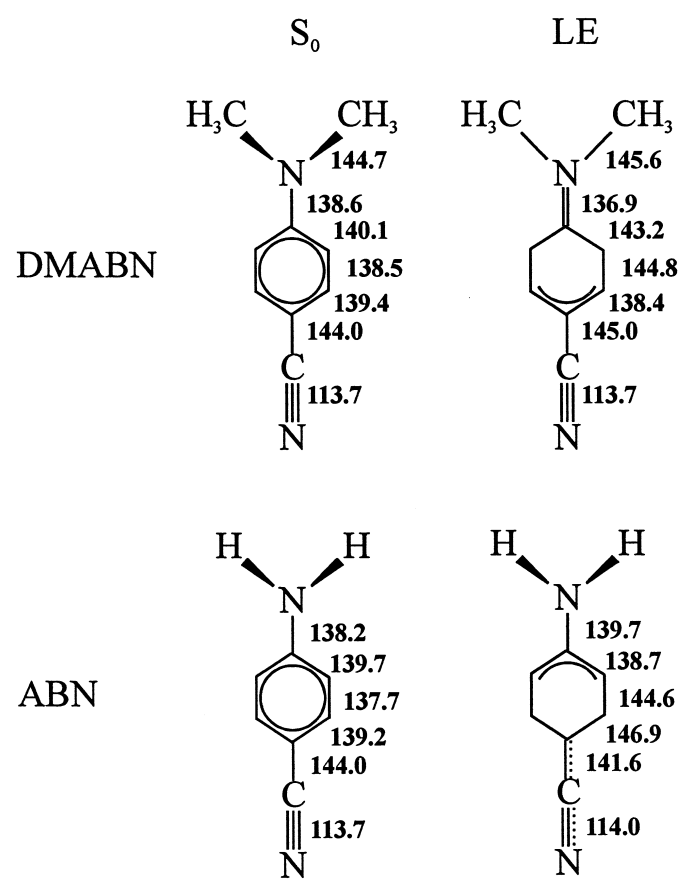

Fig. 1. Molecular structures for DMABN and ABN in ground $\left(\mathrm{S}_{0}\right)$ and LE states. Bond lengths are given in pm as obtained from CASSCF $(4,4) / 6-31 G(d)$ calculations.

Direct access to structural changes upon excitation is gained from vibrational spectroscopy. The phenyl-amino $\nu(\mathrm{PhN})$ and cyano $\nu(\mathrm{CN})$ stretching modes are expected to play a decisive role in distinguishing different models for excited-state structures by reflecting the most prominent geometry changes.

Three different models have been proposed for the structure of the CT state besides exciplex formation [18]. According to the twist ICT (TICT) model, the amino group in the CT state has a perpendicular conformation with respect to the benzene plane [2-4]. The amino group is decoupled from the benzonitrile moiety and thus the phenyl-amino bond is expected to expand leading to a lower vibrational frequency for the $\nu(\mathrm{PhN})$ mode. The pseudo-Jahn-Teller model assumes the formation of a planar CT (PICT) state with quinoidal conformation [6-9]. The phenylamino bond gains partial double bond character entailing an increased $\nu(\mathrm{PhN})$ vibrational frequency. Finally the rehybridized ICT (RICT) model implies the conversion of the cyano triple bond into a bent double bond [19]. According to calculations the $\nu(\mathrm{CN})$ vibrational frequency is expected to be observed in the area around $1600 \mathrm{~cm}^{-1}$ [19].

Nanosecond infrared (IR) [20] and resonance Raman [21,22] experiments on DMABN have been reported. From nanosecond studies, a downshift of $120 \mathrm{~cm}^{-1}$ was observed for the cyano stretching mode $\nu(\mathrm{CN})$, which is attributed to the formation of the CT state. Technical difficulties and an insufficient time resolution prevented the determination of absolute band intensity changes and their temporal evolution. Consequently, femtosecond time resolution is essential to separate the LE and CT spectra and to avoid excessive accumulation of triplet state population.

Here we present the first femtosecond time-resolved UV pump mid IR probe experiments on DMABN and ABN. Transient IR spectra are obtained for the LE states of ABN and DMABN as well as for the CT state of DMABN. In order to connect the experimental observations with structural changes, the IR spectra are modeled by ab initio CASSCF calculations. This allows the identification of the LE structures and provides the possibility to rule out certain $\mathrm{CT}$ structures.

\section{Experimental section}

The third harmonic of a regeneratively amplified Ti:Sapphire laser served for excitation $(270 \mathrm{~nm}$, $\left.\sim 10 \mathrm{~mJ} \mathrm{~cm}^{-2}\right)$. Mid-infrared (MIR) pulses $(\sim 30$ $\left.\mu \mathrm{J} \mathrm{cm}^{-2}\right)$ were generated by parametric generation and amplification followed by difference frequency mixing in a $\mathrm{AgGaS}_{2}$ crystal as described earlier [23]. The MIR pulses were tuned between 4.2 and 8.3 $\mu \mathrm{m}$. The pump-probe cross-correlation width varied between 270 and 370 fs. In Fig. 2, the time-integrated cross-correlation function is shown (dots) which was determined by probing the MIR absorption of an electron-hole plasma generated with the $270 \mathrm{~nm}$ pulses.

Fully deuterated acetonitrile $\mathrm{CD}_{3} \mathrm{CN}$ (Aldrich) was used for experiments in the frequency range of 1270 to $1690 \mathrm{~cm}^{-1}$, whereas undeuterated acetonitrile has lower absorption in the frequency range of 2000 to $2230 \mathrm{~cm}^{-1}$. Both pump and probe pulse 

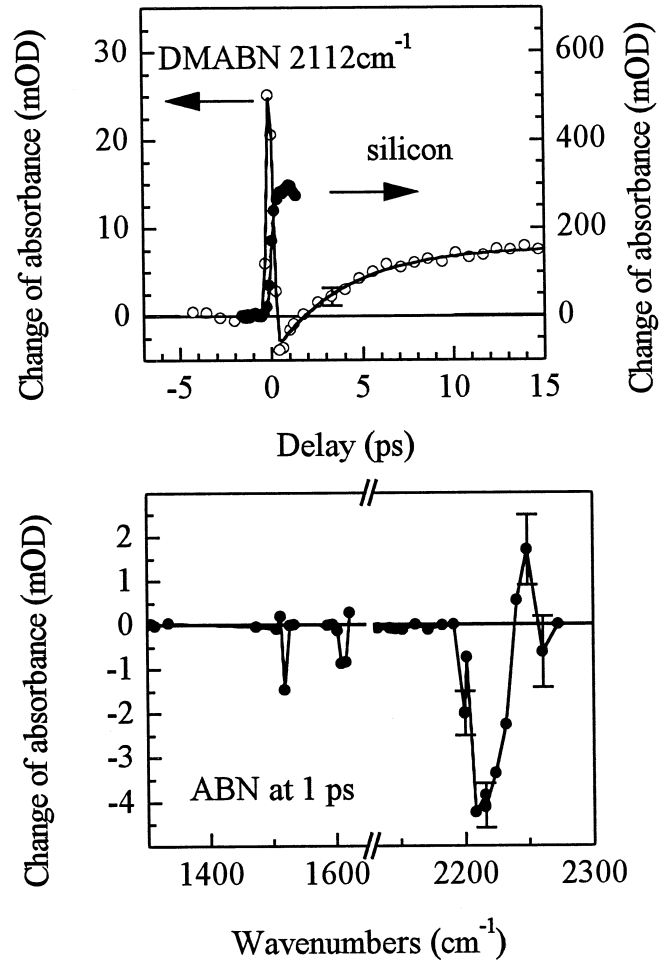

Fig. 2. Top: Change of absorbance as function of pump-probe delay time for DMABN in acetonitrile (open circles); the response function for silicon is used to determine the time resolution (320 fs, dots). Bottom: spectrum of IR absorbance change of ABN at 1 ps delay derived from individual transients.

were focused into the free streaming jet (thickness, $300 \mu \mathrm{m})$ of the sample solution $\left(5 \times 10^{-3} \mathrm{M}\right)$. The frequency-resolved absorption changes in the MIR were measured as a function of the time delay between pump and probe. A typical example is given in the top of Fig. 2 for DMABN. Differential absorption spectra were derived from such individual transients recorded for different spectral positions (Fig. 2, bottom for ABN). The molar extinction coefficient $\varepsilon_{E X}^{I R}$ of the excited states was derived from the measured change of the absorbance $\Delta \mathrm{OD}$ by

$\varepsilon_{\mathrm{EX}}^{\mathrm{IR}}=\varepsilon_{0}^{\mathrm{IR}}+N_{\mathrm{A}} \Delta \mathrm{OD} / N$

where $\varepsilon_{0}^{\mathrm{IR}}$ is the extinction coefficient of the ground state, $N_{\mathrm{A}}$ is the Avogadro constant, and $N$ is the density of excited molecules, which was calculated from the intensity of the pump pulse and the UV absorption of the jet.

\section{Computational methods}

Geometry optimizations and vibrational analyses have been performed by means of the CASSCF /6$31 \mathrm{G}(\mathrm{d})$ method implemented in the program package GAUSSIAN 94/98 [24]. In order to keep the computational effort in reasonable limits, the active space has been restricted to the two highest occupied and unoccupied $a_{2}\left(d^{\prime \prime}\right)$ and $b_{1}\left(a^{\prime}\right)$ orbitals in the $\mathrm{C}_{2 \mathrm{v}}\left(\mathrm{C}_{\mathrm{s}}\right)$ point group for non-twisted structures. For $90^{\circ}$ twisted conformations (TICT) the amino nitrogen lone-pair orbital $\left(b_{2}\left(a^{\prime \prime}\right)\right)$ has been added. Single-point calculations with extended active spaces as well as results from previously reported calculations reveal that this small active space is fully sufficient to warrant a qualitatively correct description of the electronic wave function and thus also of the geometry. IR spectra were calculated numerically. Frequencies were scaled by a factor of 0.9 , and the numerically calculated intensities by a factor of 3 for $A B N$ and 4 for DMABN (cf., Fig. 5). A more detailed presentation of theoretical results will be given elsewhere [25].

\section{Results from IR spectroscopy}

Ground-state and transient IR spectra for $A B N$ and DMABN are shown in Figs. 3 and 4, respectively. The IR lines are assigned to normal modes according to the results of calculated spectra (vide infra). Transient spectra were recorded 1 ps after excitation and can, therefore, be assigned to the LE states. For $\mathrm{ABN}$, only negligible frequency shifts and relatively small changes in the amplitudes of the IR absorption bands are observed upon excitation, which indicates that only moderate structural changes take place in the excited state (Fig. 3). Temporal profiles of the IR absorption bands do not exhibit any dynamics on a picosecond timescale. For DMABN, the transient IR spectrum of the LE state is strikingly different from that of ABN (Fig. 4). The amplitudes 
of the $\nu(\mathrm{PhN})$ and $\nu(\mathrm{CN})$ stretching modes vanish. In addition, a new band appears at $2112 \mathrm{~cm}^{-1}$ with a time constant of $4.0 \pm 0.5$ ps (Fig. 2, top). A somewhat longer time of 6 ps has previously been estimated from picosecond fluorescence decay measurements [9]. The band shifts down by $103 \mathrm{~cm}^{-1}$, in fair agreement with the downshift of $120 \mathrm{~cm}^{-1}$ observed before in an experiment with nanosecond time resolution [20].

The temporal profile of the $\nu(\mathrm{CN})$ absorption band shows induced transparency in the first $1.5 \mathrm{ps}$ (Fig. 2, top). The center frequency and width of the band are constant within $\pm 1 \mathrm{~cm}^{-1}$ for all delay times and the ultimate band intensity is slightly larger as for the $\nu(\mathrm{CN})$ mode in the ground state.

Moderate changes in the band intensities for the $\delta\left(\mathrm{CH}_{3}\right)$ and $\nu(\mathrm{Ph})$ modes are observed without frequency shift. The transients do not show dynamics
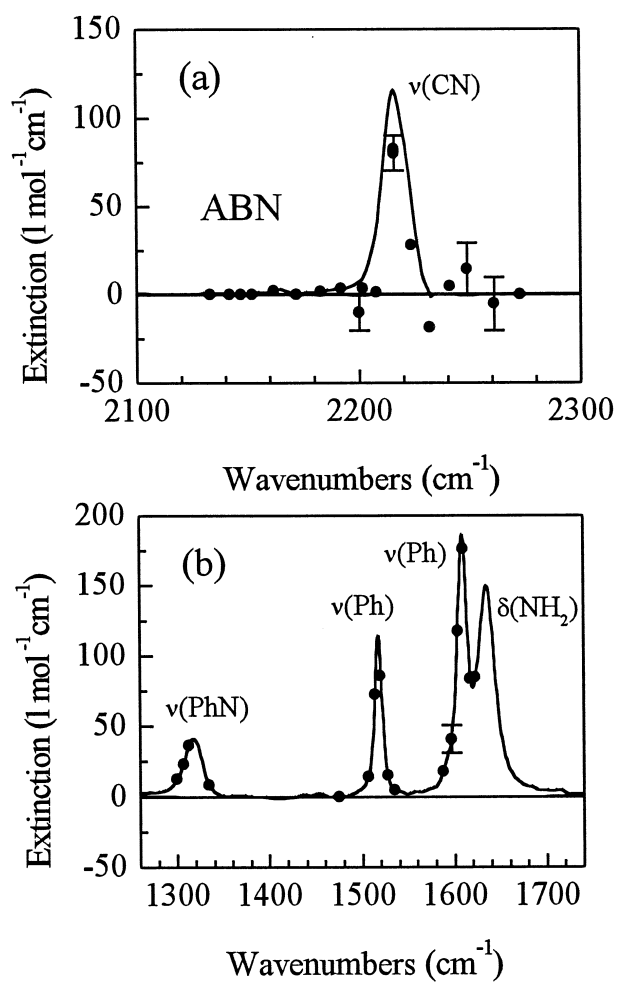

Fig. 3. Ground-state IR absorption spectrum of ABN (solid line) and absorption of excited molecules taken at 1 ps delay assigned to the LE state (dots): (a) measured in undeuterated acetonitrile, (b) in acetonitrile- $d 3$. The spectral dynamics are complete after 1 ps. Lines are marked according to calculations.
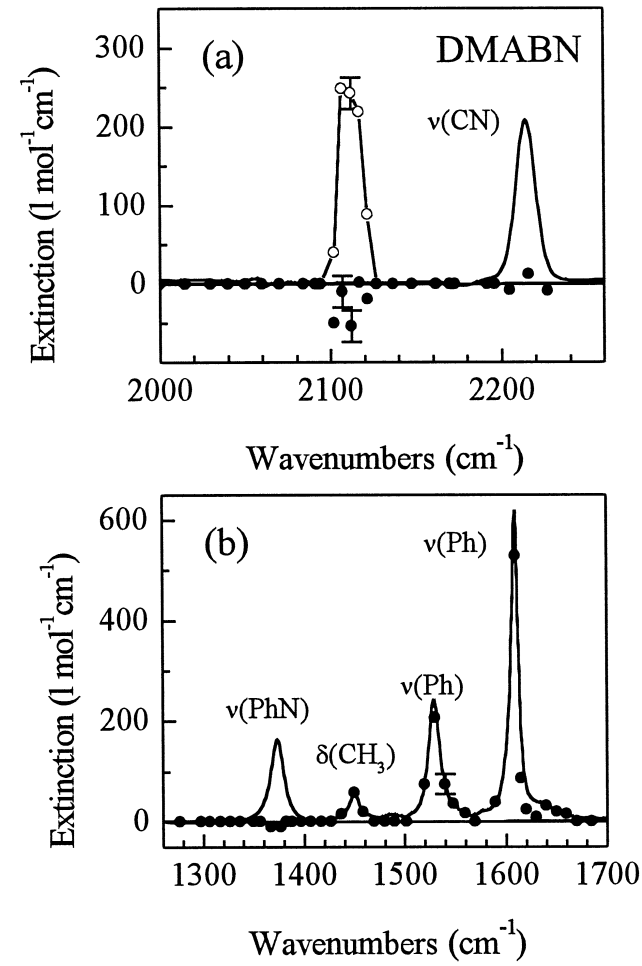

Fig. 4. Ground-state IR absorption spectrum of DMABN (solid line) and absorption of excited molecules taken at 1 ps delay assigned to the LE state (dots): (a) in undeuterated acetonitrile, (b) in acetonitrile- $d 3$. The peak at $2112 \mathrm{~cm}^{-1}$, measured at $15 \mathrm{ps}$ delay, is assigned to the CT state (open circles).

with 4 ps time constant. This rules out an accidental coincidence of the frequency of the $\nu(\mathrm{PhN})$ in the CT state with those of the $\delta\left(\mathrm{CH}_{3}\right)$ and $\nu(\mathrm{Ph})$ modes. No additional bands emerge above $1270 \mathrm{~cm}^{-1}$. Thus, the extinction coefficient of the $\nu(\mathrm{PhN})$ mode in the CT state must be much smaller than in the ground state, preventing a measurement of the vibrational frequency.

\section{Discussion}

The dynamic feature observed for the $\nu(\mathrm{CN})$ mode of DMABN allows to gain some insight into the ICT reaction. The observed 4 ps time constant 
reflects the dynamics of conversion from the LE to the CT state. The IR absorption builds up on a timescale noticeable longer than the correlation time of solvent fluctuations, which is less than $0.5 \mathrm{ps}$ for undeuterated acetonitrile [26]. Solvation dynamics are thus not expected to play a rate-determining role in this process.

Experimental results and CASSCF calculations for the ground and LE states of ABN and DMABN are compiled in Fig. 5. The corresponding geometries are depicted in Fig. 1. Excellent agreement is obtained for the frequencies of the electronic ground states with good consistency for band intensities. The calculated $\nu(\mathrm{CN})$ stretching frequency is somewhat too high, which results from underestimating the $\mathrm{C} \equiv \mathrm{N}$ bond lengths, as concluded from a comparison with crystal structure data [27]. This does, however, not affect the relative band shifts of the excited states with respect to those of the ground state. The calculated $\mathrm{LE}$ vibrational spectrum of $\mathrm{ABN}$ (Fig. 5a) shows only minor changes in line positions and band intensities as compared to the ground state, in accordance with the experimental observation. The prominent feature in the LE IR spectrum of DMABN is the strong decrease of the band intensities of both the $\nu(\mathrm{CN})$ and the $\nu(\mathrm{PhN})$ mode. These characteristics are reproduced by the calculation (Fig. 5c). Experimentally no additional lines are observed around $1500 \mathrm{~cm}^{-1}$. The calculation obviously slightly overestimates the intensity of IR lines involving methyl group deformation modes $\left(\delta\left(\mathrm{CH}_{3}\right)\right)$ in combination with benzene stretching motions $(\nu(\mathrm{Ph}))$.

In the calculations, the different characteristics of the two LE states result from different molecular structures. Whereas the geometry of the ground states and the LE state of ABN are calculated to be pyramidal, the LE state of DMABN is essentially planar (Fig. 1). A planar structure of the LE state of DMABN does not support the conclusion, derived from time-resolved fluorescence experiments [6-9],
$\mathrm{ABN}$
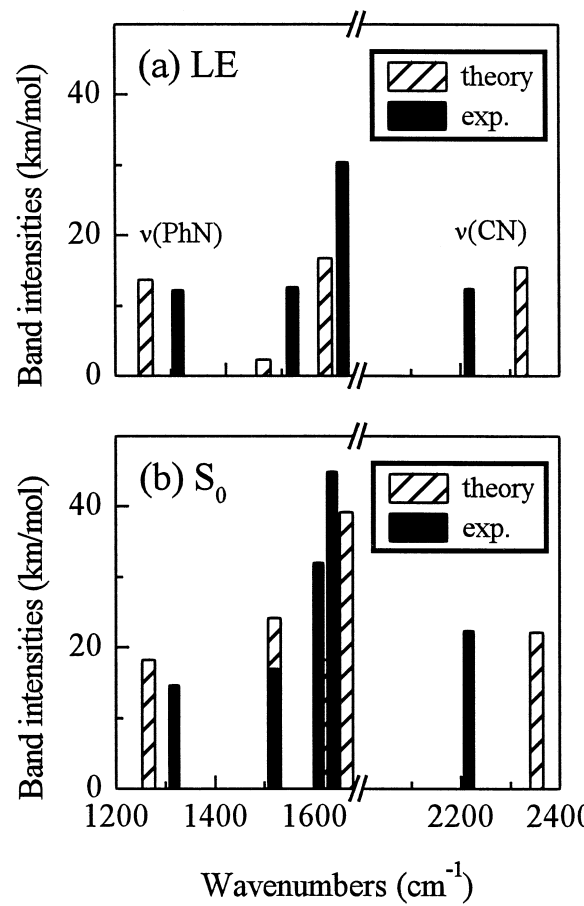

DMABN
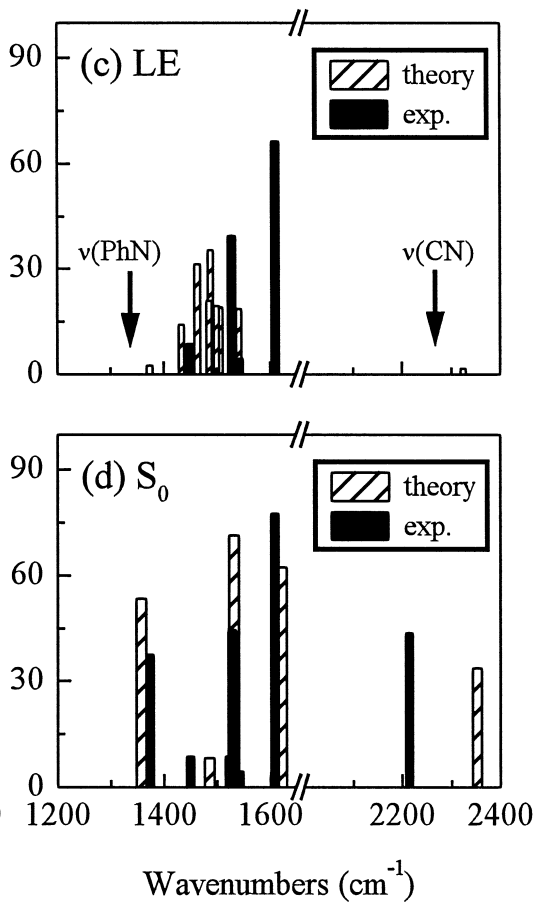

Fig. 5. Comparison of experimental and calculated IR band intensities: ABN in the (a) LE and (b) ground states; DMABN in the (c) LE and (d) ground states. 
that planarization of the amino group is the crucial reaction coordinate in the reaction of the LE to the CT state. A similar conclusion was already reached by recent ab initio calculations [10].

Computational results have also been obtained for the three structurally different CT states proposed in the literature, the TICT, PICT and RICT models. In accordance with previous calculations, the $\nu(\mathrm{CN})$ mode of the CT state in the RICT model is predicted to be substantially downshifted $\left(\approx 800 \mathrm{~cm}^{-1}\right)$ as compared to the ground state [19]. Our experimental observation is in clear contradiction with the RICT model, which hence can definitely be ruled out as valid $\mathrm{CT}$ mechanism for DMABN.

Calculated $\nu(\mathrm{CN})$ shifts of $-110 \mathrm{~cm}^{-1}$ for the TICT and $-37 \mathrm{~cm}^{-1}$ for the PICT model as well as the intensity changes are both in the same order of magnitude as the experimental shift of $-103 \mathrm{~cm}^{-1}$. A discrimination between the TICT and PICT reaction path could be made by observing the shift of the $\nu(\mathrm{PhN})$ mode frequency. Unfortunately, the IR band intensity of the $\nu(\mathrm{PhN})$ mode in the CT state is too small to be detected with the current sensitivity of the experimental set-up and will be the subject of future research.

\section{Conclusions}

Transient vibrational spectroscopy, in connection with CASSCF calculations, gives new insight into molecular structures of excited states. The LE states of $\mathrm{ABN}$ and DMABN exhibit different IR characteristics and thus have different molecular configurations, which are investigated on the basis of CASSCF calculations. This reveals that relatively minor changes in the nature of the amino substituents have a considerable impact on the excited state structure of the aminobenzonitriles putting the usefulness of aminobenzonitrile derivatives as model compounds for ICT processes in question. Although the RICT mechanism can be ruled out, an unequivocal distinction between the TICT and PICT models requires additional experimental information. Coherent Raman measurements may provide additional insight in this respect.

\section{Acknowledgements}

We thank Mrs. G. Kordass for measuring linear infrared spectra. J.D. is grateful for a fellowship of the 'Deutsche Forschungsgemeinschaft'.

\section{References}

[1] E. Lippert, W. Lüder, F. Moll, W. Nägele, H. Boos, H. Prigge, I. Seibold-Blankenstein, Angew. Chem. 73 (1961) 695.

[2] K. Rotkiewicz, K.H. Grellmann, Z.R. Grabowski, Chem. Phys. Lett. 19 (1973) 315.

[3] E. Lippert, W. Rettig, V. Bonacic-Koutecký, F. Heisel, J.A. Miehé, Adv. Chem. Phys. 68 (1987) 1.

[4] G. Köhler, K. Rechthaler, K. Rotkiewicz, W. Rettig, Chem. Phys. 207 (1996) 85.

[5] L.W. Peng, M. Dantus, A.H. Zewail, K. Kemnitz, J.M. Hicks, K.B. Eisenthal, J. Phys. Chem. 91 (1987) 6162.

[6] U. Leinhos, W. Kühnle, K.A. Zachariasse, J. Phys. Chem. 95 (1991) 2013.

[7] W. Schuddeboom, S.A. Jonker, J.H. Warman, U. Leinhos, W. Kühnle, K.A. Zachariasse, J. Phys. Chem. 96 (1992) 10809.

[8] Y.V. Il'ichev, W. Kühnle, K.A. Zachariasse, J. Phys. Chem. A 102 (1998) 5670.

[9] K.A. Zachariasse, M. Grobys, Th. von der Haar, A. Hebecker, Y.V. Il'ichev, Y.-B. Jiang, O. Morawski, W. Kühnle, J. Photochem. Photobiol. A: Chem. 102 (1996) 59.

[10] W. Sudholt, A.L. Sobolewski, W. Domcke, Chem. Phys. 240 (1999) 9, and references cited therein.

[11] G. Berden, J. van Rooy, W.L. Meerts, K.A. Zachariasse, Chem. Phys. Lett. 278 (1997) 373.

[12] O. Kajimoto, H. Yokoyama, Y. Ooshima, Y. Endo, Chem. Phys. Lett. 179 (1991) 455.

[13] V.H. Grassian, J.A. Warren, E.R. Bernstein, J. Chem. Phys. 90 (1989) 3994.

[14] U. Lommatzsch, B. Brutschy, Chem. Phys. 234 (1998) 35.

[15] A. Broo, M.C. Zerner, Theor. Chim. Acta 99 (1995) 383.

[16] A.-D. Gorse, M. Persquer, J. Phys. Chem. 99 (1995) 4039.

[17] P. Gedeck, S. Schneider, J. Photochem. Photobiol. A: Chem. 105 (1997) 165.

[18] P.C.M. Weisenborn, A.H. Huizer, C.A.G.O. Varma, Chem. Phys. 133 (1989) 437.

[19] A.L. Sobolewski, W. Sudholt, W. Domcke, J. Phys. Chem. A 102 (1998) 2716.

[20] M. Hashimoto, H. Hamaguchi, J. Phys. Chem. 99 (1995) 7875.

[21] G.D. Scholes, D. Phillips, I.R. Gould, Chem. Phys. Lett. 266 (1997) 521

[22] G.D. Scholes, I.R. Gould, A.W. Parker, D. Phillips, Chem. Phys. 234 (1998) 21.

[23] F. Seifert, V. Petrov, M. Woerner, Opt. Lett. 19 (1994) 2009. 
[24] GAUSSIAN98 (Revision A.5), M.J. Frisch, G.W. Trucks, H.B. Schlegel, G.E. Scuseria, M.A. Robb, J.R. Cheeseman, V.G. Zakrzewski, J.A. Montgomery, R.E. Stratmann, J.C. Burant, S. Dapprich, J.M. Millam, A.D. Daniels, K.N. Kudin, M.C. Strain, O. Farkas, J. Tomasi, V. Barone, M. Cossi, R. Cammi, B. Mennucci, C. Pomelli, C. Adamo, S. Clifford, J. Ochterski, G.A. Petersson, P.Y. Ayala, Q. Cui, K. Morokuma, D.K. Malick, A.D. Rabuck, K. Raghavachari, J.B. Foresman, J. Cioslowski, J.V. Ortiz, B.B. Stefanov, G. Liu, A. Liashenko, P. Piskorz, I. Komaromi, R. Gomperts, R.L.
Martin, D.J. Fox, T. Keith, M.A. Al-Laham, C.Y. Peng, A. Nanayakkara, C. Gonzalez, M. Challacombe, P.M.W. Gill, B.G. Johnson, W. Chen, M.W. Wong, J.L. Andres, M. Head-Gordon, E.S. Replogle, J.A. Pople, Gaussian, Pittsburgh, PA, 1998.

[25] J. Dreyer et al., in preparation.

[26] W.P. deBoeij, M.S. Pshenichnikov, D.A. Wiersma, J. Phys. Chem. 100 (1996) 11806.

[27] A. Heine, R. Herbst-Irmer, D. Stalke, W. Kühnle, K.A. Zachariasse, Acta Cryst. B 50 (1994) 363 